\title{
Optically tunable and detectable magnetoelectric effects in the composite consisting of magnetic thin films and InGaN/GaN multiple quantum wells
}

\author{
Ju-Ying Chen ${ }^{1}$, Yan-Ting Liou ${ }^{1}$, Chih-Ming Wei ${ }^{1}$, Sheng-Hung Chen ${ }^{1}$, \\ Minn-Tsong Lin ${ }^{* 1,2}$, Yang-Fang Chen*1 \\ ${ }^{1}$ Department of Physics, National Taiwan University, Taipei 10617, Taiwan \\ ${ }^{2}$ Institute of Atomic and Molecular Sciences, Academia Sinica, Taipei 10617, Taiwan \\ yfchen@phys.ntu.edu.tw \\ mtlin@phys.ntu.edu.tw
}

\begin{abstract}
An optically tunable and detectable magnetoeletric (ME) effect has been discovered in the composite consisting of InGaN/GaN multiple quantum wells and magnetostrictive ferromagnetic $\mathrm{Ni}$ or $\mathrm{FeCo}$ thin films at room temperature. Due to the interactively optical and piezoelectric properties of nitride semiconductors, this composite provides an intriguing optically accessible system, in which the magnetoelectric effect can be both easily tuned and detected. The underlying mechanism can be well accounted for by the interplay among magnetostrictive, piezoelectric and optical transition. It thus offers a new paradigm to generate artificial material systems with magnetic/electric/optical inter-related/controllable properties.
\end{abstract}

(C) 2013 Optical Society of America

OCIS codes: (230.3810) Magneto-optic systems.

\section{References and links}

1. M. Fiebig, "Revival of the magnetoelectric effect," J. Phys. D: Appl. Phys. 38, R123 (2005).

2. H. Zheng, J. Wang, S. E. Lofland, Z. Ma, L. Mohaddes-Ardabili, T. Zhao, L. Salamanca-Riba, S. R. Shinde, S. B. Ogale, F. Bai, D. Viehland, Y. Jia, D. G. Schlom, M. Wuttig, A. Roytburd, and R. Ramesh, "Multiferroic $\mathrm{BaTiO}_{3}-\mathrm{CoFe}_{2} \mathrm{O}_{4}$ nanostructures," Science 303, 661-663 (2004).

3. F. Zavaliche, H. Zheng, L. Mohaddes-Ardabili, S. Y. Yang, Q. Zhan, P. Shafer, E. Reilly, R. Chopdekar, Y. Jia, P. Wright, D. G. Schlom, Y. Suzuki, and R. Ramesh, "Electric field-induced magnetization switching in epitaxial columnar nanostructures," Nano Letters 5, 1793-1796 (2005).

4. Y. Zhang, C. Deng, J. Ma, Y. Lin, and C.-W. Nan, "Enhancement in magnetoelectric response in $\mathrm{CoFe}_{2} \mathrm{O}_{4}-\mathrm{BaTiO}_{3}$ heterostructure," Appl. Phys. Lett. 92, 062911 (2008).

5. T. Morita, M. K. Kurosawa, and T. Higuchi, "Cylindrical micro ultrasonic motor utilizing bulk lead zirconate titanate (PZT),” Jpn. J. Appl. Phys. 38, 3347-3350 (1999).

6. H. Ohno, D. Chiba, F. Matsukura, T. Omiya, E. Abe, T. Dietl, Y. Ohno, and K. Ohtani, "Electric-field control of ferromagnetism," Nature 408, 944-946 (2000).

7. W.-G. Wang, M. Li, S. Hageman, and C. L. Chien, "Electric-field-assisted switching in magnetic tunnel junctions," Nat Mater 11, 64-68 (2012).

8. S. Nakamura, M. Senoh, N. Iwasa, and S. ichi Nagahama, "High-brightness InGaN blue, green and yellow lightemitting diodes with quantum well structures," Jpn. J. Appl. Phys. 34, L797-L799 (1995).

9. S. P. Shuji Nakamura and G. Fasol, "The blue laser diode. the complete story," Measurement Science and Technology 12, 755 (2001). 
10. S. Nakamura, M. Senoh, S. ichi Nagahama, N. Iwasa, T. Yamada, T. Matsushita, H. Kiyoku, and Y. Sugimoto, "InGaN multi-quantum-well-structure laser diodes with cleaved mirror cavity facets," Jpn. J. Appl. Phys. 35, L217-L220 (1996).

11. T. Palacios, A. Chakraborty, S. Heikman, S. Keller, S. DenBaars, and U. Mishra, "AlGaN/GaN high electron mobility transistors with ingan back-barriers," IEEE Electron Device Lett. 27, 13 - 15 (2006).

12. F. Bernardini and V. Fiorentini, "Spontaneous versus piezoelectric polarization in III - V nitrides: Conceptual aspects and practical consequences," phys. stat. sol. (b) 216, 391-398 (1999).

13. S. F. Chichibu, A. C. Abare, M. S. Minsky, S. Keller, S. B. Fleischer, J. E. Bowers, E. Hu, U. K. Mishra, L. A. Coldren, S. P. DenBaars, and T. Sota, "Effective band gap inhomogeneity and piezoelectric field in InGaN/GaN multiquantum well structures," Appl. Phys. Lett. 73, 2006-2008 (1998).

14. T. Y. Lin, "Converse piezoelectric effect and photoelastic effect in InGaN/GaN multiple quantum wells," Appl. Phys. Lett. 82, 880-882 (2003).

15. C. H. Chen, W. H. Chen, Y. F. Chen, and T. Y. Lin, "Piezoelectric, electro-optical, and photoelastic effects in $\mathrm{In}_{\mathrm{x}} \mathrm{Ga}_{1-\mathrm{x}} \mathrm{N} / \mathrm{GaN}$ multiple quantum wells," Appl. Phys. Lett. 83, 1770-1772 (2003).

16. H. Hong, K. Bi, and Y. Wang, "Magnetoelectric performance in $\mathrm{Ni} / \mathrm{Pb}(\mathrm{Zr}, \mathrm{Ti}) \mathrm{O}_{3} / \mathrm{FeCo}$ trilayered cylindrical composites," J. Alloys Comp. 545, 182 - 185 (2012).

17. N. Vernier, D. A. Allwood, D. Atkinson, M. D. Cooke, and R. P. Cowburn, "Domain wall propagation in magnetic nanowires by spin-polarized current injection," EPL 65, 526 (2004).

18. A. G. Kontos, Y. S. Raptis, N. T. Pelekanos, A. Georgakilas, E. Bellet-Amalric, and D. Jalabert, "Micro-raman characterization of $\mathrm{In}_{\mathrm{x}} \mathrm{Ga}_{1-\mathrm{x}} \mathrm{N} / \mathrm{GaN} / \mathrm{Al}_{2} \mathrm{O}_{3}$ heterostructures," Phys. Rev. B 72, 155336 (2005).

19. C. H. Chen, W. H. Chen, Y. F. Chen, and T. Y. Lin, "Piezoelectric, electro-optical, and photoelastic effects in $\mathrm{In}_{\mathrm{x}} \mathrm{Ga}_{1-\mathrm{x}} \mathrm{N} / \mathrm{GaN}$ multiple quantum wells," Appl. Phys. Lett. 83, 1770-1772 (2003).

20. H. J. Chang, Y. P. Hsieh, T. T. Chen, Y. F. Chen, C.-T. Liang, T. Y. Lin, S. C. Tseng, and L. C. Chen, "Strong luminescence from strain relaxed $\mathrm{InGaN} / \mathrm{GaN}$ nanotips for highly efficient light emitters," Opt. Express 15, 9357-9365 (2007).

21. T. Suzuki, H. Baba, and E. Matsumoto, "Stress effect on hysteretic magnetization curve of nickel," Int. J. Appl. Electromag. and Mech. 13, 307-310 (2002).

22. C. M. Wei, H. Y. Shih, Y. F. Chen, and T. Y. Lin, "Optical detection of magnetoelectric effect in the composite consisting of ingan/gan multiple quantum wells and FeCo thin film,” Appl. Phys. Lett. 98, 131913 (2011).

\section{Introduction}

Magnetoelectric (ME) effect based on the composites consisting of magnetostrictive and piezoelectric materials is defined as the mutual induction of an electric field and a magnetic field. When an external magnetic field is applied to the composite, the magnetic field causes the magnetostrictive material to strain. Meanwhile, this deformation can transfer to the piezoelectric material, and generates an electric field. Conversely, an external electric field can induce a strain in the piezoelectric material, which deforms the magnetostrictive material and causes the change in magnetic permeability. Due to the ME effect, piezoelectric/magnetostrictive composite materials possess a great deal of potential applications, including modulation of amplitudes, polarizations and phases of optical waves, ME data storage and switching; spin-wave generation, amplification and frequency conversion, etc [1]. They thus play an important role in modern emerging technologies.

So far, many investigations have shown that perovskite ceramics and magnetic oxides are excellent piezoelectric materials for the synthesis of ME composites [2, 3, 4]. For example, $\mathrm{PbZr}_{1-\mathrm{x}} \mathrm{Ti}_{\mathrm{x}} \mathrm{O}_{3}(\mathrm{PZT}) / \mathrm{Tb}_{1-\mathrm{x}} \mathrm{Dy}_{\mathrm{x}} \mathrm{Fe}_{2}$ ) alloys (Terfenol-D) and PZT/ferrite have been widely studied with regard to investigating ME effects [1]. However, bulk PZT has a disadvantage that ceramic materials are not suitable for microfabrication processes, such that there exists a great hurdle for the realization of practical application [5].

Here, we propose a new composite system consisting of InGaN/GaN multiple quantum wells (MQWs) and ferromagnetic thin films, and this composite system offers a different path from the previously reported piezoelectric/magnetostrictive materials $[6,7]$. Due to the correlated optical and piezoelectric properties of nitride semiconductors, this composite system reveal the ME effect at room temperature, which can be easily detected optically and also possess the optically tunable characteristics. The finding of the optical accessibility and controllability of 


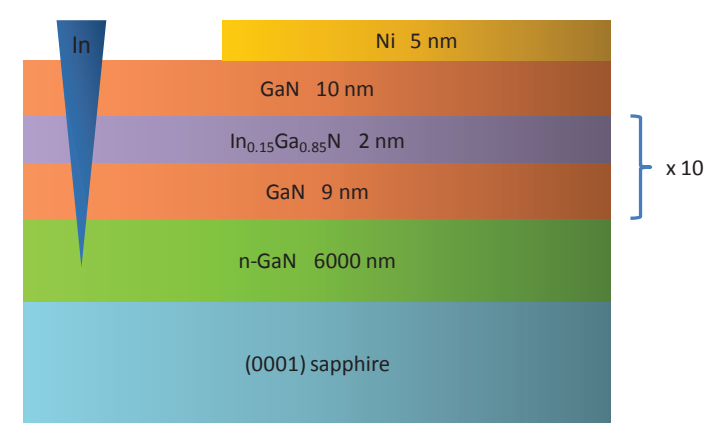

Fig. 1. The schematic structure of InGaN/GaN MQWs and Ni thin film

this design can extend the conventional ME systems to integrate with mature optoelectronic devices. Our approach thus provides a new paradigm to create artificial material systems with magnetic/electric/optical inter-related/controllable properties.

As well known, InGaN/GaN MQWs are very important III-V semiconductors. Due to the tunability of the energy gap, they have been widely used for the fabrication of light emitting devices $[8,9]$, laser diodes $[8,9,10]$ ranging from infrared to ultraviolet light spectra, and high electron mobility transistors [11]. In addition, they possess a wurtzite structure with an excellent piezoelectricity. Because of the inherent nature of lattice mismatch, nitride heterostructures exhibit a large spontaneous piezoelectric polarization and built-in internal electric field $[12,13$, $14,15]$.

In this work, we used two distinct features to demonstrate the ME effect in the composites consisting of InGaN/GaN MQWs and magnetostrictive ferromagnetic Ni and FeCo thin films. Rather than measuring the ME voltage coefficient directly, we monitor the change of magnetic properties in the composite via applying an external electric field or an external excitation light source. Besides, we also optically detect the change of piezoelectric (PZ) properties in the composite via applying an external magnetic field. Since the PZ field strongly depends on the surface deformation of InGaN/GaN MQWs, the magnetoelectric effect can thus be easily achieved based on the new composite of InGaN/GaN MQWs and magnetostrictive ferromagnetic thin films. Furthermore, since the PZ field in InGaN/GaN MQWs can be manipulated by varying optical excitation power, ME effects thus become optically tunable in this novel system.

\section{Experiment}

InGaN/GaN MQWs as shown in figure 1 were prepared by low temperature metal-organic chemical vapor deposition. An undoped series of ten periods of 2 -nm-thick $\operatorname{In}_{0.15} \mathrm{Ga}_{0.85} \mathrm{~N}$ well and 9-nm-thick GaN barriers were grown on (0001) sapphire. There is a $6 \mu \mathrm{m} \mathrm{n}$-GaN layer between the sapphire and the MQWs. The contact of $n-G a N$ is made by indium deposition and the annealing temperature is at $300^{\circ} \mathrm{C}$. The other contact of undoped $\mathrm{GaN}$ is a $\mathrm{Ni}$ or $\mathrm{FeCo}$ thin Film. The Ni thin film was deposited on InGaN/GaN MQWs by the evaporation, with a base pressure lower than $5 \times 10^{-7}$ Torr. The FeCo thin film was deposited with the weight percentage of $25 \%$ and $75 \%$ for Fe and Co, respectively, on the InGaN/GaN MQWs by a high vacuum magnetron sputtering system. The base pressure is lower than $1 \times 10^{-8}$ Torr and the $\mathrm{dc}$ power voltage is at $320 \mathrm{~V}$ in a $5 \times 10^{-3}$ Torr Ar ambient. 

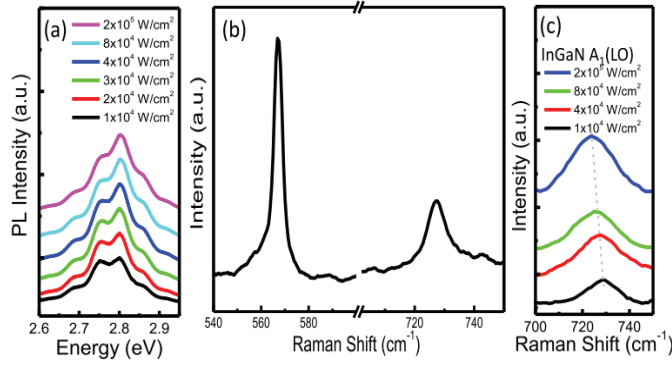

Fig. 2. (a) Photoluminescence spectra of InGaN/GaN MQWs under different excitation power density with an excitation wavelength of $325 \mathrm{~nm}$, (b) and (c) Raman scattering spectra of InGaN/GaN MQWs with an excitation wavelength of $325 \mathrm{~nm}$.

\section{Results and discussion}

The existing built-in electric field in the studied InGaN/GaN MQWs has been examined, as shown in the figure 2. We demonstrate here the existence of built-in electric field and piezoelectricity in the studied InGaN/GaN MQWs. Figure 2(a) displays the PL spectra of InGaN/GaN MQWs under optical excitation of $325 \mathrm{~nm}$ with different power density. Each spectrum is dominated by a strong emission and an obvious interference pattern due to multiple reflections within the air/nitride epilayers/sapphire system. The emission at around $2.8 \mathrm{eV}$ can be attributed to the excitonic transition of the InGaN quantum wells. It can be seen that the relative intensity between the most intense two peaks changes remarkably as the excitation power density increases. This change comes from the fact that the dominant emission shifts toward the high-energy side while the interference pattern remains at its position as the excitation power density increases. This behavior can be well understood according to the quantum-confined Stark effect(QCSE). The spontaneous and piezoelectric polarizations will induce a strong internal electric field and tilt the band alignment in InGaN/GaN MQWs. As the excitation power density increases, the screening of the internal electric field caused by photoexcited electron-hole pairs is more effective and the tilting of the band structure becomes less pronounced. Therefore, it leads to the increase in the transition energy and the blueshift in the observed spectra.

Figure 2(b) shows the $\mu$-Raman scattering spectra of InGaN/GaN MQWs by a $\mathrm{HeCd}$ laser with a wavelength of $325 \mathrm{~nm}$. There are two resolved phonon structures, in which the peak around $565 \mathrm{~cm}^{-1}$ is from the E2 mode of the strained n-GaN, and the peak around $730 \mathrm{~cm}^{-1}$ is the $\mathrm{InGaN} \mathrm{A}_{1}(\mathrm{LO})$ mode. Figure 2(c) displays the Raman scattering spectra of InGaN/GaN MQWs under different excitation power density. It is found that the frequency of the InGaN $\mathrm{A}_{1}(\mathrm{LO})$ phonon mode decreases when the excitation power density increases. This interesting behavior can be understood as follows. The built-in internal electric field will be reduced due to the screening effect caused by photoexcited electron-hole pairs. Because the nitride semiconductors are good piezoelectric materials, a change in the internal electric field can produce a strain and alter the lattice constant, which leads to the change in the Raman scattering spectra. The thermal effect caused by laser heating can be ruled out because the dependence of frequency shift on temperature has a negligible rate of $-3 \times 10^{-2} \mathrm{~cm}^{-1} / \mathrm{K}$ for $A_{1}(\mathrm{LO})$ phonons.

In order to demonstrate that the external electric field on InGaN/GaN MQWs can cause the change in the magnetic characteristics of Ni film, we measure magnetic hysteresis loops as shown in figure 3(a) at room temperature. We use the magneto-optical Kerr effect (MOKE) to monitor the magnetic response of the samples by a He-Ne laser with a wavelength of $633 \mathrm{~nm}$, which is smaller than the band gap of InGaN/GaN MQWs to avoid the effect arising from band gap absorption. The evolution in MOKE signal upon magnetization indicates that the saturated 

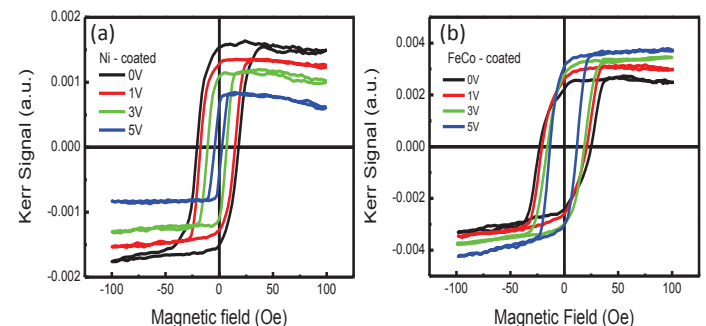

Fig. 3. (a) and (b). Hysteresis curves of Ni-coated and FeCo-coated InGaN/GaN MQWs by means of MOKE measurement with the applied magnetic field parallel to the plane of the sample under different external electric potentials.

magnetic signal and the coercive field decrease when the external electric field increases.

To understand the underlying mechanism of the above phenomenum, we replace the Ni film by a FeCo film, and measure the magnetization hysteresis loops as shown in figure 3(b). Figure 3(b) shows that the saturated magnetic signal of the FeCo thin film increases and the coercive field decreases when the external electric field is increased. Quite interestingly, the behavior of the saturated magnetic signal shows an opposite trend compared with that of the Ni film. We recall the well known fact that the magnetostrictive characteristics of $\mathrm{FeCo}$ are opposite to those of $\mathrm{Ni}$ [16]. This means that the length of $\mathrm{Ni}$ thin film will be extended along the external magnetic field, while that of $\mathrm{FeCo}$ thin film will be shortened along the external magnetic field. Therefore, the same deformation caused by $\mathrm{InGaN} / \mathrm{GaN}$ MQWs will produce an opposite trend for the magnetization of $\mathrm{Ni}$ and FeCo films, which is consistent with the result shown in figure 3. We thus draw a conclusion that the change of the magnetization of $\mathrm{Ni}$ and $\mathrm{FeCo}$ films arises from the deformation of InGaN/GaN MQWs caused by the external electric field through the piezoelectricity of nitride layers. On the contrary, the trends of the coercive fields are the same for $\mathrm{Ni}$ and $\mathrm{FeCo}$ thin films under an applied external electric field. This behavior could be attributed to the propagation of domain walls induced by the force generated by the electrical current [17]. To confirm our interpretation, we have performed the change of magnetization under light illumination without the external electric field, which can be used to distinguish the effect of electrical current. Figures 4(a) and (b) show that the saturated magnetic signal of $\mathrm{Ni}$ thin film increases and the coercive field remains constant when a $266 \mathrm{~nm}$ pulse laser is used as a light source to screen the PZ field of InGaN/GaN MQWs. This behavior can be well understood according to the well-known QCSE. The spontaneous and piezoelectric polarizations can induce a strong internal electric field and tilt the band alignment in InGaN/GaN MQWs. As the excitation power density increases, the screening of the internal electric field caused by photoexcited electron-hole pairs is more effective and the tilting of the band structure becomes less pronounced [14]. The influence of the light screening on the PZ field will induce a magnetostrictive force and cause the increase of the saturation magnetization of $\mathrm{Ni}$ thin film. Similarly, the saturation magnetization of FeCo thin film decreases. Because there is no electric current, the coercive fields of $\mathrm{Ni}$ and $\mathrm{FeCo}$ thin films remain the same. It therefore provides a concrete evidence to support our proposed mechanism that the tends of the coercive fields as shown in figure 3 are caused by the propagation of domain wall induced by the electric current. In addition, this intriguing result also demonstrates a unique feature of optically tunable ME possessed by our newly designed composite composed of ferromagnetic thin films and nitride MQWs.

To further evidence that an external electric field can introduce the change of lattice constant of InGaN/GaN MQWs through the PZ field, we have measured the Ramen scattering spectra 

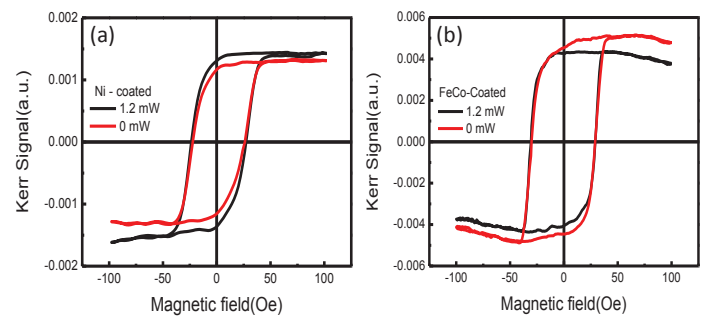

Fig. 4. (a) and (b) Hysteresis curves of Ni-coated and FeCo-coated InGaN/GaN MQWs with and without an excitation source of $266 \mathrm{~nm}$ wavelength.

using a Jobin-Yvon T64000 system with an Ar-ion laser working at $514.5 \mathrm{~nm}$ in the backscattering geometry. The incident and scattered light beams were parallel to the c axis, which in turn was normal to the growth surface. Figure 5(a) shows the $\mu$-Raman scattering spectra of the InGaN/GaN MQWs under different electric fields. The peak around $730 \mathrm{~cm}^{-1}$ is the InGaN $\mathrm{A}_{1}(\mathrm{LO})$ mode [18]. The energy of the $\mathrm{InGaN} \mathrm{A}_{1}(\mathrm{LO})$ phonon decreases when the electric field is applied [19]. The magnitude of the strain $\varepsilon$ can be estimated from the Raman scattering spectra by the expression:

$$
\varepsilon=\frac{\Delta \omega}{2\left(\mathrm{a}-\mathrm{b} \frac{\mathrm{C}_{13}}{\mathrm{C}_{33}}\right)},
$$

where $\Delta \omega$ is the shift in the $\mathrm{InGaN}_{1}(\mathrm{LO})$ phonon mode, $\mathrm{a}$ and $\mathrm{b}$ are phonon deformation potentials, and $\mathrm{C}_{13}$ and $\mathrm{C}_{33}$ stand for elastic constants [20]. The value of $\mathrm{a}, \mathrm{b}, \mathrm{C}_{13}$, and $\mathrm{C}_{33}$ were obtained by the extrapolation of the data for $\mathrm{GaN}$ and $\mathrm{InN}$ [18]. According to the above equation and the frequency of $\mathrm{InGaN}_{1}(\mathrm{LO})$ phonon mode in figure 5(a), the strain of InGaN in the InGaN/GaN MQWs can be calculated. The compressive strain of InGaN in the InGaN/GaN MQWs decreases when the applied electric field increases. In other words, when the external electric field increases, the $\mathrm{Ni}$ and FeCo thin films experience an in-plane tensile strain. This tensile strain can lead to the decrease of the magnetization of the Ni thin film and the increase of the magnetization of the FeCo thin film [21]. This is the reason why the saturated magnetic signal of Ni thin film decreases and that of FeCo thin film increases when the external electric field increases. Figure 5(b) shows that the calculated strain and the Kerr signal have a linear relationship, which is consistent with the previous experimental and theoretical results within the elastic limit [21]. It implies that the change of the magnetization of $\mathrm{Ni}$ and FeCo thin films caused by the deformation of crystalline lattice arises from the PZ effect of InGaN/GaN MQWs.

Next, for the completeness of the investigation of the ME effect, we demonstrate that an external magnetic field applied on a magnetostrictive material can generate an electric field in InGaN/GaN MQWs as shown in our preliminary work [22]. We use a $10 \mathrm{~nm}$ FeCo thin film as the magnetostrictive material deposited on InGaN/GaN MQWs. By measuring the PL spectra of the FeCo-coated InGaN/GaN MQWs under different applied magnetic fields and light excitation with a wavelength of $325 \mathrm{~nm}$, we found that, as shown in figure 6(a), a strong emission dominates each spectrum and an obvious interference pattern caused by multiple reflections within the air/nitride epilayers/sapphire system appears. The dominant emission peak shows a blue shift in the PL spectra when the applied magnetic field increases. Furthermore, there exists a critical magnetic field at about 300 Gauss, beyond which the peak feature gradually saturates. A similar phenomenon is found in $\mu$-Raman scattering spectra of the FeCo-coated InGaN/GaN MQWs under different applied magnetic fields, as shown in figure 7(a). The frequency of In$\mathrm{GaN}_{1}(\mathrm{LO})$ phonon mode decreases when the magnetic field increases, and saturates when the magnetic field exceeds the critical magnetic field at about 300 Gauss, similar to the result 

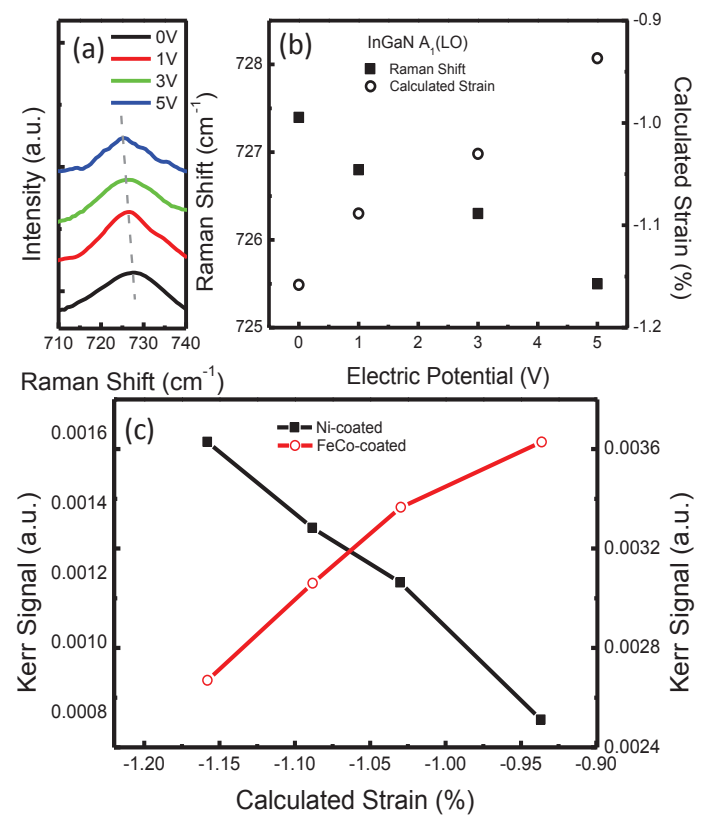

Fig. 5. (a) Raman scattering spectra of the InGaN/GaN MQWs under different external electric potentials. (b) The Kerr Signal of Ni-coated and FeCo-coated InGaN/GaN MQWs as a function of calculated strain.

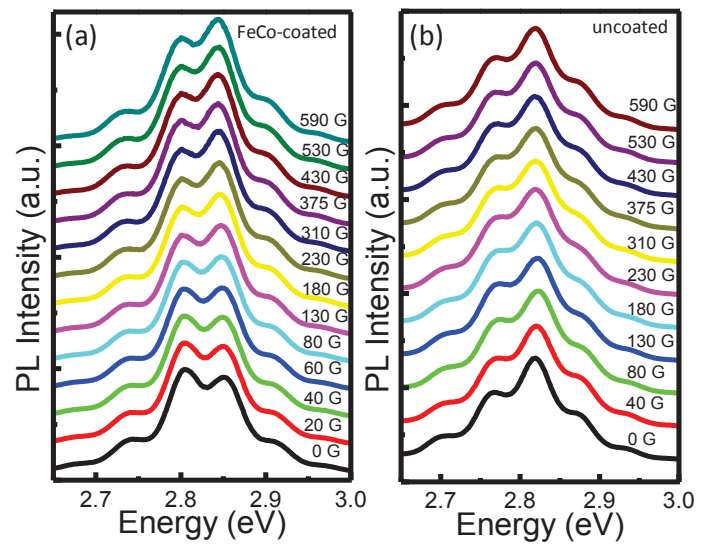

Fig. 6. Photoluminescence spectra of (a) FeCo-coated and (b) uncoated InGaN/GaN MQWs under different applied magnetic field. [22]

in figure 6(a). On the contrary, in uncoated InGaN/GaNMQWs, figures 6(b) and 7(b) show that such PL and Raman shifts do not appear under different applied magnetic fields.

Figure 6(c) shows the peak position of the InGaN $A_{1}(\mathrm{LO})$ phonon mode frequency and the calculated strain as a function of applied magnetic field. When the external magnetic field increases, the strain of the FeCo-coated InGaN/GaN MQWs decreases. Moreover, the calculated strain also gradually saturates beyond the 300 Gauss threshold. The inset in figure 7(c) shows the hysteresis curve of the FeCo-coated InGaN/GaN MQWs obtained by MOKE measurement with the applied magnetic field parallel to the plane. The coercivity is about 160 Gauss and the 

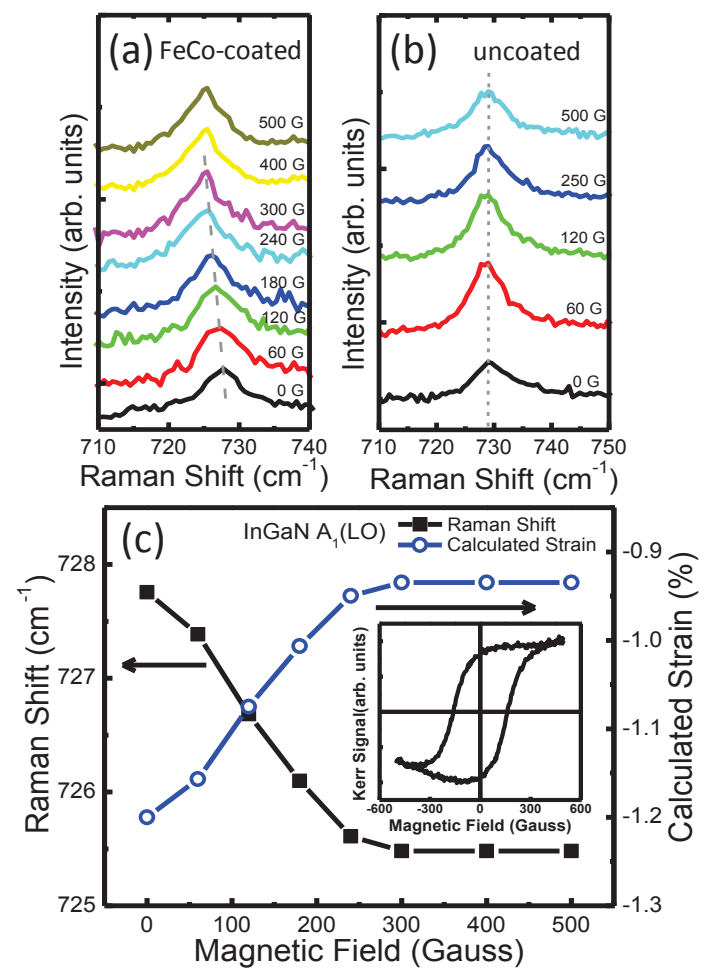

Fig. 7. Raman scattering spectra of (a) FeCo-coated and (b) uncoated InGaN/GaN MQWs under different applied magnetic field. (c) Frequencies of $\operatorname{InGaN~}_{1}(\mathrm{LO})$ phonon mode in FeCo-coated InGaN/GaN MQWs and the calculated strain as a function of applied magnetic field. The inset shows the hysteresis curve of FeCo-coated InGaN/GaN MQWs with the applied magnetic field parallel to the sample plane. [22]

saturation field is about 300 Gauss. The shifts in the PL spectra, the $\mu$-Raman scattering spectra, and the calculated strain shown in figures 6(a),7(a), and 7(c) share a very similar saturation behavior in term of the saturation field, as shown in the inset of figure 7(c). This result indicates the strong correlation among the blueshifts in the PL spectra, the redshifts in the InGaN $\mathrm{A}_{1}(\mathrm{LO})$ phonon frequency, the strain in the InGaN/GaN MQWs, and the magnetization of the FeCo thin film.

The ME effect can be used to explain the above phenomena very well. The FeCo thin film deforms along the applied magnetic field due to the magnetostriction. This deformation transfers to the piezoelectric layers, the InGaN/GaN MQWs, and generates an electric polarization. Then, the induced electric polarization screens the built-in internal electric field and reduces the compressive strain, which reduces the tilting of the band structure of InGaN/GaN MQWs. Therefore, the PL peak blueshifts and the frequency of the InGaN $A_{1}(\mathrm{LO})$ phonon redshifts. When the applied magnetic field exceeds the saturation field, the magnetization remains constant and in turn the magnetostrictive effect saturates. As a result, the deformation and the optical properties of InGaN/GaN MQWs follow the same trend.

\section{Conclusion}

In summary, we have demonstrated the ME effect in new composites consisting of InGaN/GaN multiple quantum wells and magnetostrictive ferromagnetic thin films. The ME effect is very 
pronounced and can be detected optically at room temperature. In addition, the ME effect can be manipulated by the optical transition within multiple quantum wells and becomes optically tunable. Based upon both advantages of optical detection and tunability, the composites shown here pocesses a unique feature of multi-functionality, which can combine the novel ME effects and outstanding optical and piezoelectric characteristics of nitride semiconductors. The new composite shown here represents an excellent illustration for the generation of artifitial material system with magnetic/electric/optical inter-related/controllable properties. In addition, it provides a new route to integrate ME effects with mature light emitting technology, which holds a wider and an immediate scientific impact and is able to produce distinct devices for practical applications in the near future.

\section{Acknowledgments}

This work was supported by the National Science Council and the Ministry of Education of Taiwan. 